\title{
Long term monitoring of the optical background in the Capo Passero deep-sea site with the NEMO tower prototype
}

\author{
S. Adrián-Martínez ${ }^{20}$, S. Aiello ${ }^{4}$, F. Ameli ${ }^{9}$, M. Anghinolfi ${ }^{6}$, M. Ardid ${ }^{20}$, G. Barbarino ${ }^{7,15}$, E. Barbarito ${ }^{1,11}$,

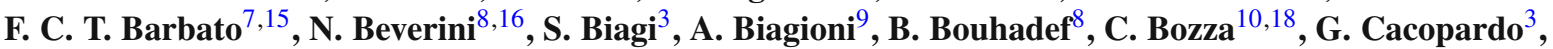 \\ M. Calamai ${ }^{8,16}$, C. Calì ${ }^{3}$, D. Calvo ${ }^{21}$, A. Capone ${ }^{9,17}$, F. Caruso $^{3}$, A. Ceres ${ }^{1}$, T. Chiarusi ${ }^{2}$, M. Circella ${ }^{1}$, \\ R. Cocimano ${ }^{3}$, R. Coniglione ${ }^{3}$, M. Costa ${ }^{3}$, G. Cuttone ${ }^{3}$, C. D’Amato ${ }^{3}$, A. D'Amico ${ }^{3,23}$, G. De Bonis ${ }^{9}$, V. De Luca ${ }^{3}$, \\ N. Deniskina ${ }^{7}$, G. De Rosa ${ }^{7,15}$, F. di Capua ${ }^{7,15}$, C. Distefano ${ }^{3}$, A. Enzenhöfer ${ }^{22}$, P. Fermani ${ }^{9}$, G. Ferrara ${ }^{3,13}$,

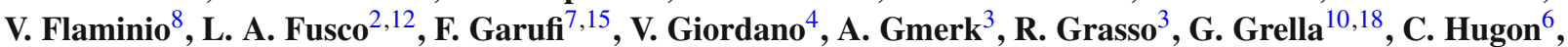 \\ M. Imbesi ${ }^{3}$, V. Kulikovskiy ${ }^{3}$, R. Lahmann ${ }^{22}$, G. Larosa ${ }^{3}$, D. Lattuada ${ }^{3}$, K. P. Leismüller ${ }^{3}$, E. Leonora ${ }^{4}$, P. Litrico ${ }^{3}$, \\ C. D. Llorens Alvarez ${ }^{20}$, A. Lonardo ${ }^{9}$, F. Longhitano ${ }^{4}$, D. Lo Presti ${ }^{4,13}$, E. Maccioni ${ }^{8,16}$, A. Margiotta ${ }^{2,12}$, \\ A. Marinelli ${ }^{8,16}$, A. Martini ${ }^{5}$, R. Masullo ${ }^{9,17}$, P. Migliozzi $^{7}$, E. Migneco ${ }^{3}$, A. Miraglia ${ }^{3}$, C. M. Mollo ${ }^{7}$, M. Mongelli ${ }^{1}$,

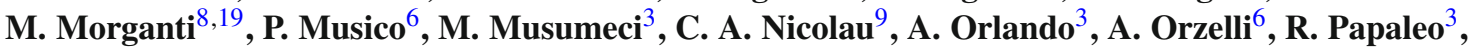

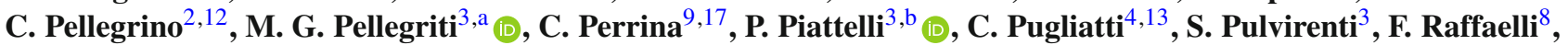 \\ N. Randazzo $^{4}$, D. Real ${ }^{21}$, G. Riccobene ${ }^{3}$, A. Rovelli ${ }^{3}$, M. Saldaña ${ }^{20}$, M. Sanguineti ${ }^{6}$, P. Sapienza ${ }^{3}$, V. Sciacca ${ }^{3}$,

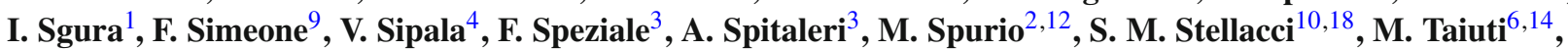

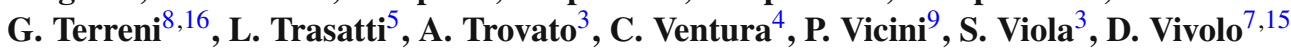 \\ ${ }^{1}$ INFN Sezione Bari, Via E. Orabona 4, 70126 Bari, Italy \\ 2 INFN Sezione Bologna, V.le Berti Pichat 6/2, 40127 Bologna, Italy \\ ${ }^{3}$ INFN Laboratori Nazionali del Sud, Via S.Sofia 62, 95123 Catania, Italy \\ ${ }^{4}$ INFN Sezione Catania, Via S. Sofia 64, 95123 Catania, Italy \\ 5 INFN Laboratori Nazionali di Frascati, Via Enrico Fermi 40, 00044 Frascati, Italy \\ ${ }^{6}$ INFN Sezione Genova, Via Dodecaneso 33, 16146 Genoa, Italy \\ ${ }^{7}$ INFN Sezione Napoli, Via Cintia, 80126 Naples, Italy \\ 8 INFN Sezione Pisa, Polo Fibonacci, Largo Bruno Pontecorvo 3, 56127 Pisa, Italy \\ ${ }^{9}$ INFN Sezione Roma, P.le A. Moro 2, 00185 Rome, Italy \\ 10 INFN Gruppo Collegato di Salerno, Via Giovanni Paolo II 132, 84084 Fisciano, Italy \\ ${ }^{11}$ Dipartimento Interateneo di Fisica Università di Bari, Via E. Orabona 4, 70126 Bari, Italy \\ 12 Dipartimento di Fisica ed Astronomia Università di Bologna, V.le Berti Pichat 6/2, 40127 Bologna, Italy \\ 13 Dipartimento di Fisica e Astronomia Università di Catania, Via S. Sofia 64, 95123 Catania, Italy \\ ${ }^{14}$ Dipartimento di Fisica Università di Genova, Via Dodecaneso 33, 16146 Genoa, Italy \\ 15 Dipartimento di Scienze Fisiche Università di Napoli, Via Cintia, 80126 Naples, Italy \\ ${ }^{16}$ Dipartimento di Fisica Università di Pisa, Polo Fibonacci, Largo Bruno Pontecorvo 3, 56127 Pisa, Italy \\ ${ }^{17}$ Dipartimento di Fisica Università "Sapienza", P.le A. Moro 2, 00185 Rome, Italy \\ ${ }^{18}$ Dipartimento di Fisica Università di Salerno, Via Giovanni Paolo II 132, 84084 Fisciano, Italy \\ ${ }^{19}$ Accademia Navale di Livorno, viale Italia 72, 57100 Livorno, Italy \\ ${ }^{20}$ Instituto de Investigación para la Gestión Integrada de las Zonas Costeras, Universitat Politècnica de València, Gandia, Spain \\ ${ }^{21}$ IFIC-Instituto de Física Corpuscular, CSIC-Universitat de València, Valencia, Spain \\ ${ }^{22}$ Erlangen Centre for Astroparticle Physics, Friedrich-Alexander-Universität Erlangen-Nürnberg, Erlangen, Germany \\ 23 Present Address: Nikhef, Science Park, Amsterdam, The Netherlands
}

Received: 20 July 2015 / Accepted: 26 January 2016 / Published online: 9 February 2016

(C) The Author(s) 2016. This article is published with open access at Springerlink.com

\begin{abstract}
The NEMO Phase-2 tower is the first detector which was operated underwater for more than 1 year at the "record" depth of $3500 \mathrm{~m}$. It was designed and built within the framework of the NEMO (NEutrino Mediterranean Obser-
\end{abstract}

\footnotetext{
a e-mail: pellegriti@lns.infn.it

b e-mail: piattelli@lns.infn.it
}

vatory) project. The $380 \mathrm{~m}$ high tower was successfully installed in March 201380 km offshore Capo Passero (Italy). This is the first prototype operated on the site where the Italian node of the KM3NeT neutrino telescope will be built. The installation and operation of the NEMO Phase-2 tower has proven the functionality of the infrastructure and the operability at $3500 \mathrm{~m}$ depth. A more than 1 year long monitoring 
of the deep water characteristics of the site has been also provided. In this paper the infrastructure and the tower structure and instrumentation are described. The results of long term optical background measurements are presented. The rates show stable and low baseline values, compatible with the contribution of ${ }^{40} \mathrm{~K}$ light emission, with a small percentage of light bursts due to bioluminescence. All these features confirm the stability and good optical properties of the site.

\section{Introduction}

High energy neutrinos are expected to be messengers from astrophysical objects where hadronic interactions may take place. In this framework several projects were developed (see Refs. [1-3]). The discovery of a cosmic neutrino flux reported by IceCube initiated the era of Neutrino Astronomy. This result, at first based on an analysis performed selecting cascade contained events or track events starting in the detector (the so called HESE analysis) [4,5], was confirmed by the observation of a neutrino cosmic flux of upgoing muon neutrinos [6]. These fluxes are obtained with independent analyses in two different sets of events and show rather close values when normalized to one flavor. However, most of the questions raised about the origin of the observed neutrino cosmic flux remain unsolved. Many hypotheses have been proposed including galactic and extragalactic sources, Dark Matter etc. As a consequence of this discovery, the physics case for the construction of $\mathrm{a} \mathrm{km}^{3}$-scale neutrino telescope in the Northern Hemisphere is strongly enforced. A telescope in the Mediterranean Sea has a field of view for up-going neutrinos, that are the golden channel for neutrino astronomy, of $87 \%$ thus covering a very large part of the of the Galactic Plane including the Galactic Centre. Observations put in evidence the presence or several gamma-ray sources in the $\mathrm{GeV}-\mathrm{TeV}$ energy range nearby the Galactic Centre. Since the origin of these emissions is unknown the observation of neutrinos from these regions can have a unique role in clarifying the production mechanisms. Moreover, the very good angular resolution achievable thanks to the large effective scattering length in deep sea water is one of the most distinct feature of a cubic kilometre telescope installed in the Mediterranean Sea and it is very important for the individuation of sources.

Since 1998, the NEMO (NEutrino Mediterranean Observatory) collaboration carried out research activities aimed at developing and validating key technologies for a $\mathrm{km}^{3}$-scale underwater neutrino telescope [7-10] as well as searching and characterizing a deep-sea site suitable for the installation of the detector. The first pilot project, NEMO Phase-1, dates back to 2007 when a prototype tower was deployed at a depth of $2000 \mathrm{~m}$ in a Test Site located about $20 \mathrm{~km}$ offshore Catania (Italy). Technical details and the scientific results can be found in [11].

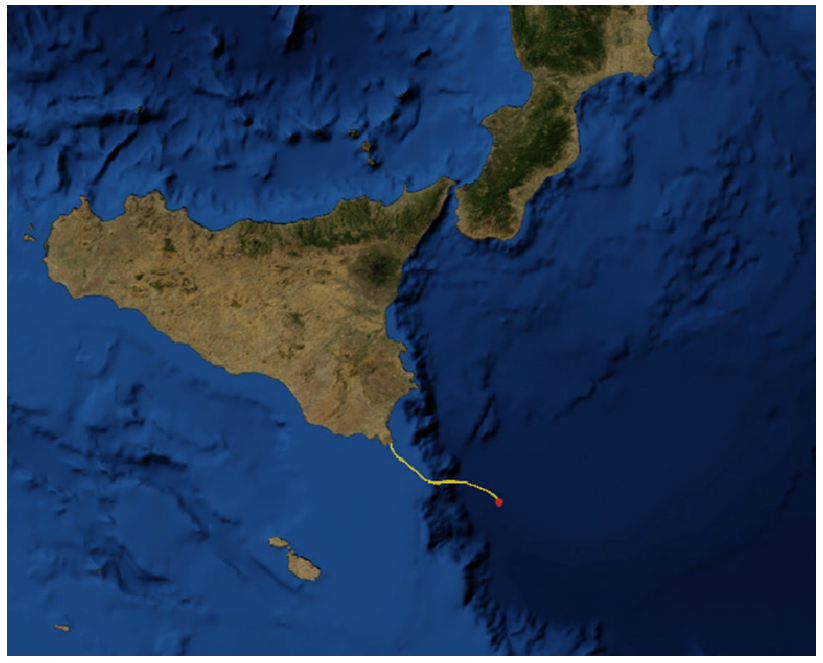

Fig. 1 Map of the western Ionian region showing the location of the KM3NeT-It deep-sea site and the electro-optical cable route

A deep-sea site at a depth of $3500 \mathrm{~m}$, about $80 \mathrm{~km}$ offshore Portopalo di Capo Passero (Italy) (36 $16^{\circ} 17^{\prime} 48^{\prime \prime} \mathrm{N}$, $15^{\circ} 58^{\prime} 42^{\prime \prime} \mathrm{E}$ ), was identified as optimal for the installation of the underwater neutrino telescope (Fig. 1). In the following it is indicated as the KM3NeT-It site.

In 2008 a $100 \mathrm{~km}$ electro-optical cable connecting the KM3NeT-It site to an on-shore station, located in Portopalo di Capo Passero, was deployed. In the following years an important $R \& D$ activity aimed at validating new technologies was carried out thanks to this infrastructure. These activities culminated in the construction, deployment and operation of the NEMO Phase-2 tower in March 2013. The Phase2 tower continuously took data until August 2014 when it was disconnected to allow for an upgrade of the underwater infrastructure in preparation for the installation of the first group of detection structures that will form the Italian node of the KM3NeT detector.

The paper is structured as follows. In Sect. 2 the Capo Passero site and infrastructure are described. Section 3 describes the detection tower and the details of all the included sub-systems. Section 4 describes the installation and the sea operations, which are necessary for the longterm measurements of the optical background presented in Sect. 5. Conclusion and perspectives are given in Sect. 6.

\section{The KM3NeT-It site and infrastructure}

The KM3NeT-It site was characterised with a series of sea campaigns to study and monitor the most important environmental parameters. Oceanographical properties of the site, like deep-sea water optical properties (absorption and diffusion), water environmental properties (temperature, salinity), biological activity, optical background, water currents, 


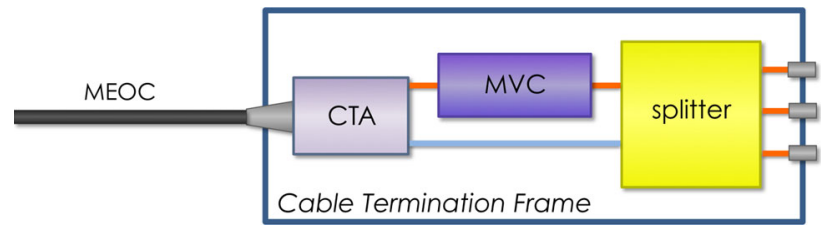

Fig. 2 Scheme of the Cable Termination Frame. Power lines and optical fibres of the Main Electro Optical Cable (MEOC) are split by a Cable Termination Assembly (CTA). The $10 \mathrm{~kW}$ DC/DC Medium Voltage Converter (MVC) steps down the voltage from $10 \mathrm{kV}$ to $375 \mathrm{~V}$. A splitter assembly reroutes power lines and optical fibres towards three wet-mateable electro-optical connectors

sedimentation and seabed nature were studied [12-14]. This activity has confirmed that the KM3NeT-It site has optimal characteristics to host the Italian node of the KM3NeT research infrastructure [15].

The KM3NeT-It infrastructure includes a shore station, a $100 \mathrm{~km}$ long main electro-optical cable (MEOC) and a deepsea installation comprising a novel design $10 \mathrm{~kW}$ DC voltage converter. The onshore infrastructure, located in Portopalo di Capo Passero, hosts the power feeding system, which directly feeds the MEOC [16], as well as the control centre and the data acquisition system.

The MEOC is a standard submarine communication cable manufactured by Alcatel ${ }^{1}$ containing 20 G655 optical fibres. ${ }^{2}$ These fibres have a maximum attenuation of $0.23 \mathrm{~dB} / \mathrm{km}$ at $1550 \mathrm{~nm}$ wavelength. The cable has a single conductor for power transmission. Current return is provided via seawater, which offers an extremely small resistance thus reducing power losses [17]. The system incorporates sea electrodes both at the shore (anode) and at the deep-sea (cathode) ends. At the deep-sea end the MEOC is terminated by a Cable Termination Frame (CTF) (Figs. 2, 3) hosting a Cable Termination Assembly (CTA), allowing to split and reroute the fibres towards a connection panel, and a DC Medium Voltage Converter (MVC) to step down the $10 \mathrm{kV}$ provided by the onshore Power Feeding System to 375 V [18]. The intermediate voltage is distributed to three output ports equipped with wet-mateable connectors. Similarly optical fibre communication is distributed to the same output ports. Connections are performed by means of a Remotely Operated Vehicle (ROV).

In the configuration in place at the time when the prototype described in this paper was operated, only eight out of the twenty optical fibres of the MEOC were unfolded and distributed to the three outputs available on the CTF. In July 2015 a new CTF that uses an MVC identical to the previous one has been successfully installed. This new CTF includes a splitter able to distribute all the twenty available fibers of the

\footnotetext{
$\overline{1}$ Model OALC-4 17 mm Type 30.

2 Corning Vascade LEAF negative dispersion NZ-DSF.
}

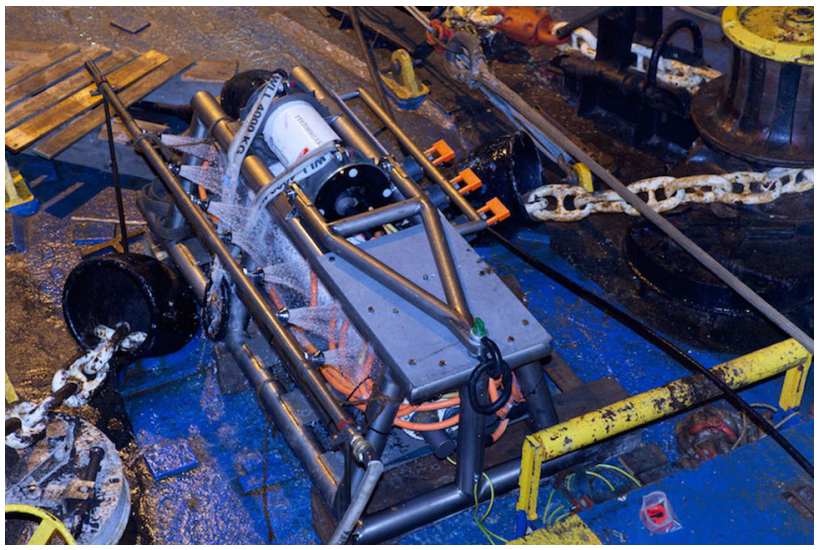

Fig. 3 An image of Cable Termination Frame on the ship deck prior to its deployment

MEOC to five electro-optical output and will be used as main distribution node of the KM3NeT-It phase-one detector.

\section{The NEMO Phase-2 tower}

The detection unit deployed in the KM3NeT-It site at a depth of $3449 \mathrm{~m}$ is a tower-like structure composed of eight horizontal elements (named floors). Each floor is a $8 \mathrm{~m}$ long marine grade aluminum structure connected to its next neighbours by means of eight tensioning ropes [19]. The arrangement of these ropes is such that each floor is forced to a position perpendicular to its vertical neighbours, as shown in Fig. 4. An iron anchor fixes the structure to the seabed while an appropriate buoyancy at the top provides the pull to keep the structure taut. The floors are vertically spaced by $40 \mathrm{~m}$, with the lowermost one positioned $100 \mathrm{~m}$ above the sea bottom. Each floor holds four Optical Modules (OM), two at each end, one looking vertically downwards and the other horizontally outwards. The structure is designed to be assembled and deployed in a compact configuration (Fig. 5) and to be unfurled once on the sea bottom under the pull provided by the buoy.

A schematics of the tower is shown in Fig. 6. In addition to the $32 \mathrm{OMs}$ the instrumentation installed includes several sensors for calibration and environmental monitoring. In particular for positioning calibration purposes two hydrophones are installed close to the ends of each floor and two others on the tower base. Being the deep seawater a dynamical medium, monitoring of its oceanographic and optical properties during the detector operation is important since they can have an impact on the detector performance. For that reason environmental probes are installed on the tower: two Conductivity-Temperature-Depth (CTD) probes, ${ }^{3}$ installed

\footnotetext{
${ }^{3}$ Sea Bird Electronics, 37-SM Micro-CAT.
} 


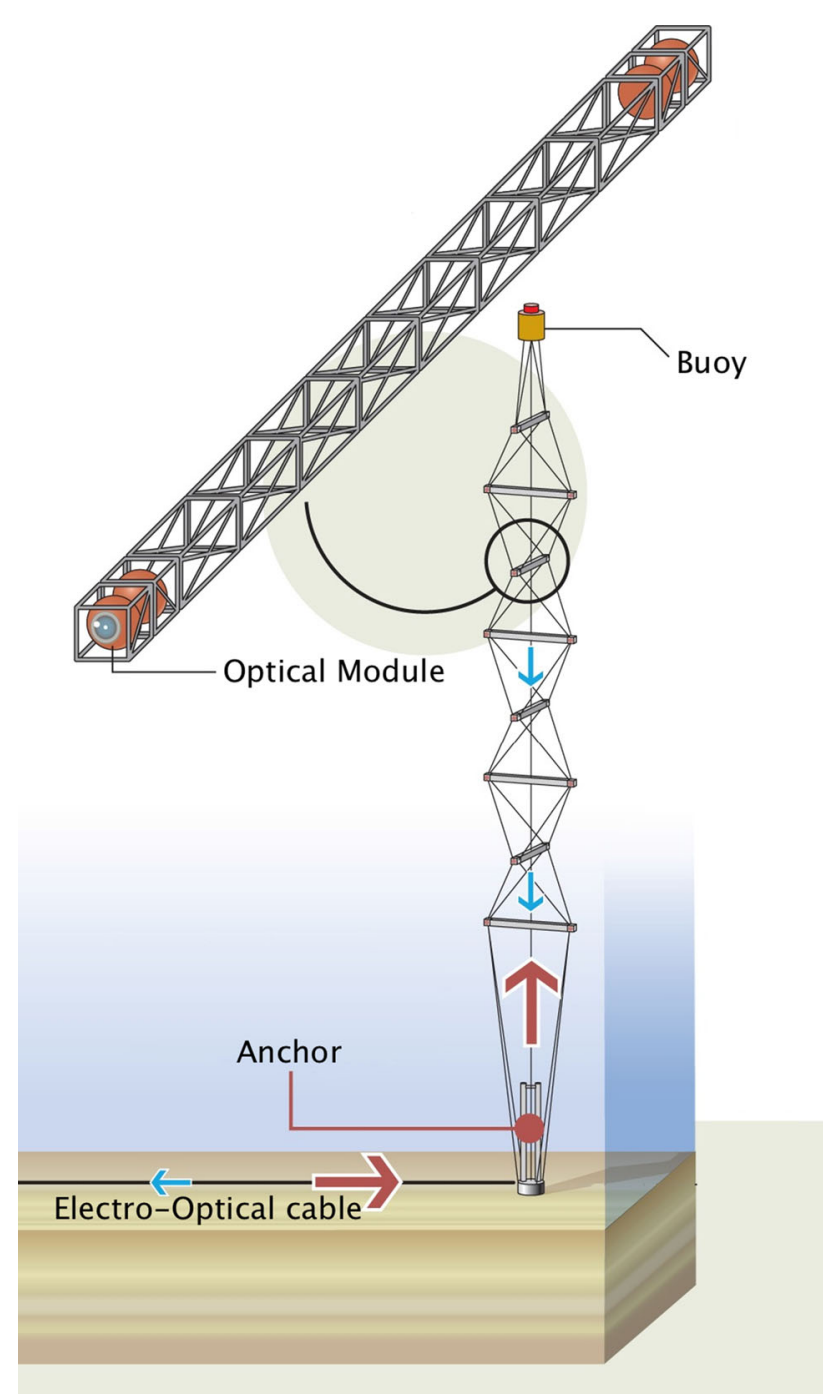

Fig. 4 Sketch of the NEMO Phase-2 tower. The structure is formed by a sequence of eight floors, each one supporting four optical modules. The structure is anchored at the seabed and kept taut by a buoy located at the top. Vertical distances are not to scale

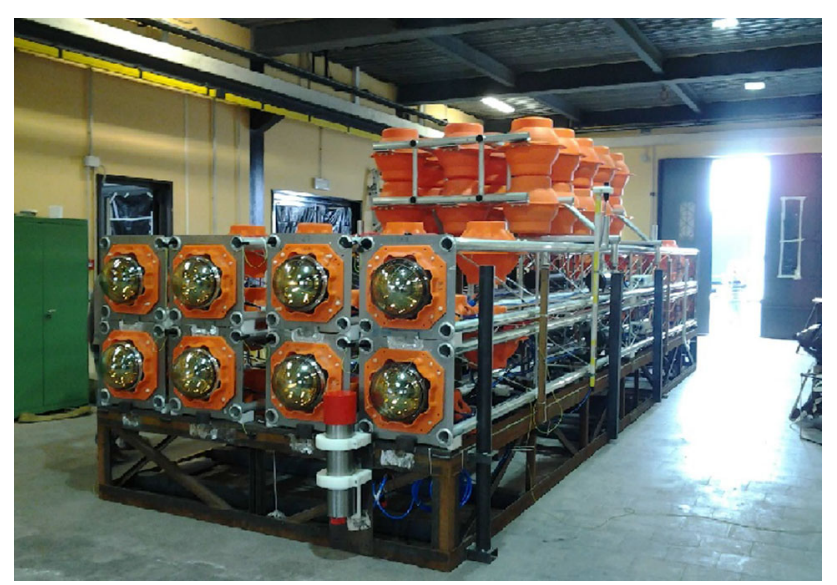

Fig. 5 The NEMO Phase-2 tower in its compact configuration during the integration phase

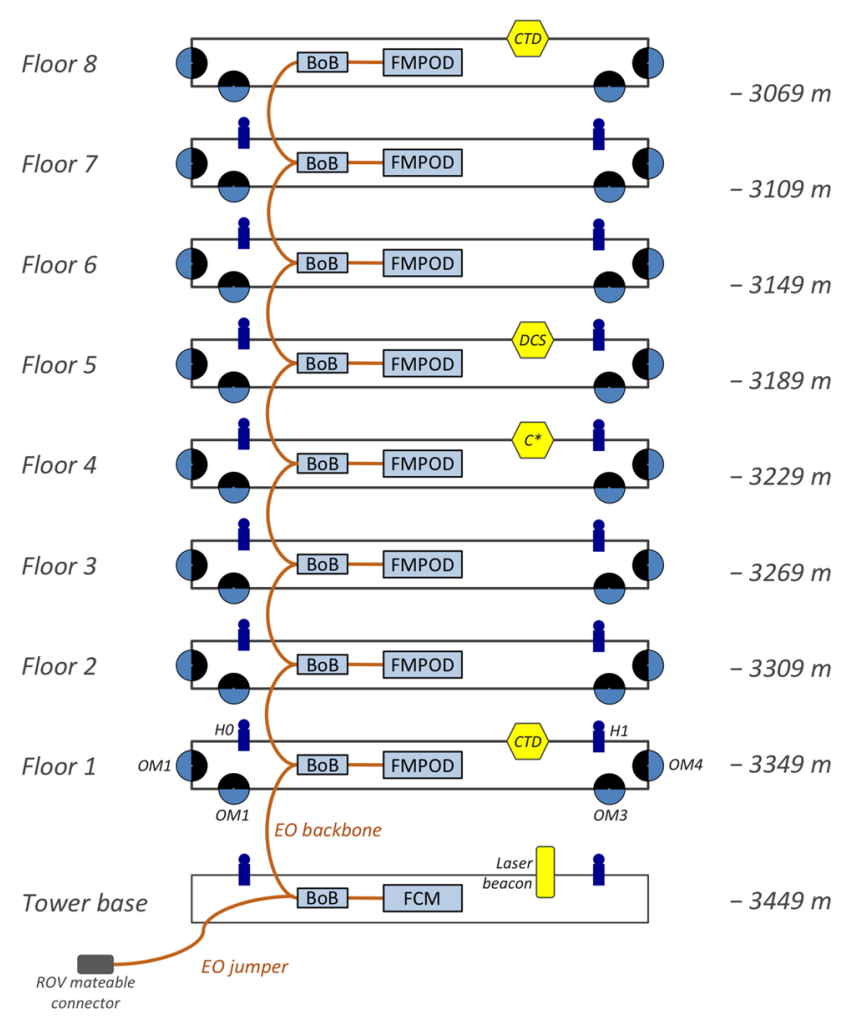

Fig. 6 Schematics of the NEMO Phase-2 tower, including the backbone cabling (orange), the Floor Module Protective Oceanic Device (FMPOD) and the electro-optical breakouts (BoB). Connection to the Junction Box is provided through a ROV-mateable hybrid connector, installed in the tower base. The position of the environmental probes are also indicated as well as those of the hydrophones ( $\mathrm{H} 0$ and $\mathrm{H} 1$ ) (see text for details). Nominal depths of each floor are given on the right

on the 1st and 7th floor; a light transmissometre used for the measurement of blue light attenuation in seawater $\left(C^{*}\right)$; a Doppler Current Sensor (DCS) used to monitor deep sea currents installed on the 5th floor.

Power distribution and data transmission along the tower is fulfilled by means of a "backbone" electro-optical cable. This is a lightweight umbilical subsea cable,${ }^{4}$ carrying 10 electrical conductors and 12 optical fibres.

\subsection{The optical modules}

The OM is the basic element of the NEMO Phase- 2 detection unit $[20,21]$. Each OM consists of a 13-inch pressure resistant (up to 700 bar) borosilicate glass sphere,,${ }^{5}$ housing a 10-inch Hamamatsu photomultiplier (PMT R7081-SEL) [22] together with its high-voltage power supply, read-out electronics and calibration system (Fig. 7). Mechanical and optical contact between the PMT and the internal glass surface is ensured by an optical silicone gel. A $\mu$-metal cage

\footnotetext{
$\overline{4}$ Nexans S.A.

5 Nautilus Marine GmbH, VITROVEX flotation sphere.
} 

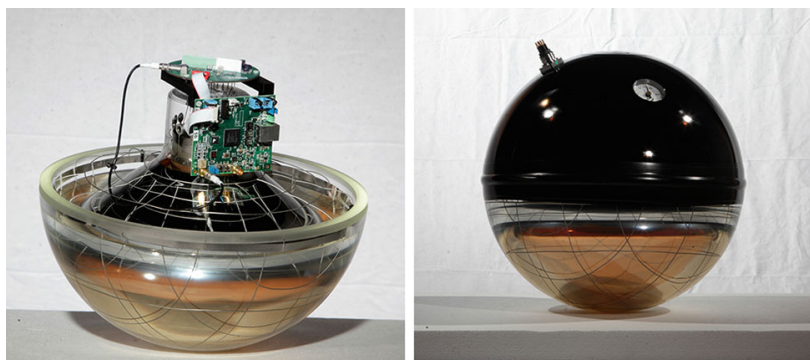

Fig. 7 Left One hemisphere of the optical module glass vessel containing the photomultiplier and the front-end module board. Right An optical module fully integrated with the electro-optical feedthrough and the pressure-meter

shields the PMT from the Earth's magnetic field. A detailed description is given in [20].

PMT signal digitization is provided by a Front-End Module board that is also housed inside the OM [23]. The board samples the analog PMT signal using two 8-bit Fast Analog to Digital Converters (Fast-ADCs) running at $100 \mathrm{MHz}$ and staggered by $5 \mathrm{~ns}$. This technique gives the desired sampling rate yet allowing for a power dissipation lower than a single $200 \mathrm{MHz}$ ADC. To match the large dynamic range of the PMT signal to the input voltage range of the ADCs, the signal is compressed by a non-linear circuit, which applies a quasi-logarithmic signal compression.

Four OMs were also equipped with a prototype RFID based system that allowed to acquire oceanographic data with readout through the glass sphere without the use of feedthroughs [24].

\subsection{Electronics and cabling}

A simplified scheme of the electronics and cabling of the tower is shown in Fig. 8. At the level of each floor the backbone is split by means of breakout boxes $(\mathrm{BoB})$. Each breakout is a high-density polyethylene vessel filled with silicone

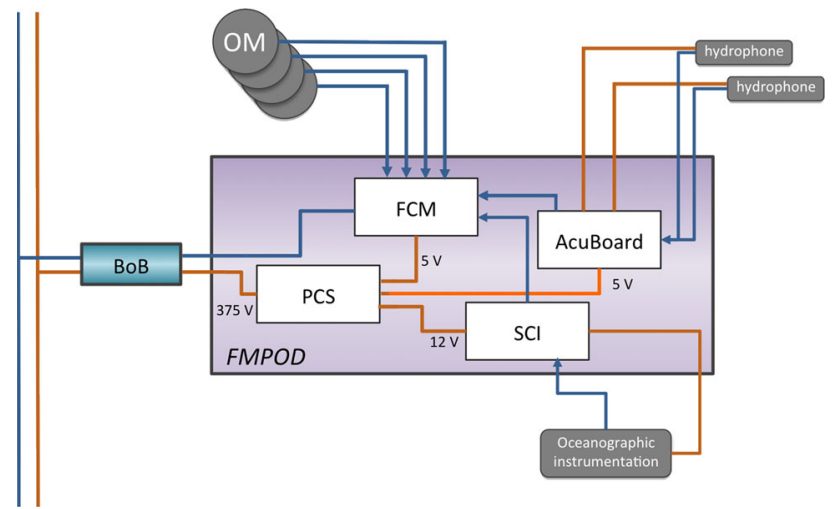

Fig. 8 Schematics of the floor system. See text for the definition of the acronyms oil and pressure compensated. The BoB is equipped with two hybrid penetrators, which are used to split the backbone, and two connectors (one electrical and one optical) from the backbone to the floor cabling system.

On each floor the four OM digital data are sent to a Floor Control Module board (FCM) housed in a pressure resistant vessel, the Floor Module Protective Oceanic Device (FMPOD). The FMPOD is fitted in the middle of the floor mechanical structure and houses the data acquisition, control and the power distribution systems of the floor. A similar setup is adopted for the tower base.

The $375 \mathrm{~V}$ supplied by the MVC is monitored at the level of the tower base and distributed to the eight floors. Inside the floor FMPOD a Power Control System board (PCS) provides conversion of the DC supply from $375 \mathrm{~V}$ to the low voltages needed by the electronics as well as monitoring and control of the main electrical parameters of the floor.

A Slow Control Interface board (SCI) provides the interface to the oceanographic instruments installed on the floor via RS-232 serial standard. In addition, each SCI has two analogue sensors to monitor humidity and temperature inside the FMPOD.

The acoustic board (AcuBoard) is a part of the positioning system described in Sect. 3.5.1.

\subsection{Data transmission system}

The link to the shore-station uses an optical fibre as physical layer and implements a high speed serial link using a proprietary data format [25]. All data are encoded into a serial $800 \mathrm{Mb} / \mathrm{s}$ stream by a serializer, converted into optical signal by an electro-optical transceiver and transmitted to the shore station. In the communication protocol the data stream is divided into $125 \mu$ s long frames of 10,000 bytes each.

A transmission system through optical links based on Dense Wavelength Division Multiplexing (DWDM) technology was chosen [26]. It is implemented by means of add-anddrop passive devices that multiplex/demultiplex many optical channels at different wavelengths into/from the same fibre. Two wavelengths are associated to each floor of the tower, one for the sea-to-shore and one for the shore-to-sea communication. The optical wavelengths are chosen according to the ITU standard grid with $100 \mathrm{GHz}$ frequency spacing in the CBand thus allowing up to 45 channels per fibre. The DWDM network provides, indeed, a point-to-point communication between the shore-station equipment and the deep-sea apparatus. Each FCM contains an add-and-drop filter that allows to add or subtract the specific optical wavelength allocated to the floor. Data from all floors are thus transmitted through the backbone in the same fibre.

Detector data are received onshore by a dedicated electronics board, named Ethernet FCM (EFCM), which is based 
on a Virtex $-5^{6}$ development board. This board collects the data received by the underwater electronics and transfers them to the DAQ and storage systems through a Gigabit Ethernet connection.

\subsection{Data acquisition system}

Thanks to the extremely large communication bandwidth available ( $2 \mathrm{Gpbs})$, no hardware triggers are implemented underwater: all the digitized signals are sent to shore. The data stream originated by each FCM is addressed on shore to the twin EFCM board, which transfers the data to the onshore Trigger and Data Acquisition System (TriDAS) [27].

Each detected photon pulse is sampled by the FEM and arranged by the FCM in a hit record with a mean size of 28 bytes. Consequently the tower averaged optical throughput is about $250 \mathrm{Mbps}$. The total amount of data from offshore includes also a stream of about $80 \mathrm{Mbps}$ from the acoustic sensors and a negligible contribution of slow control data. The electronics were designed to deal with an optical signal up to $150 \mathrm{kHz}$ continuous single rate on each PMT without dead time.

From the EFCMs, the PMT optical data-stream is routed through a $1 \mathrm{~Gb}$ Ethernet network to the first layer of the TriDAS, composed of two Hit Managers (HM) processes running on two CPUs. Each HM gathers data from half a tower and coherently time-slices the continuous stream of data into time intervals of $200 \mathrm{~ms}$. All the data corresponding to a given interval of time are sent by the HMs to a single TriggerCPU process (TCPU). Subsequent time slices are addressed to the others TCPU processes, implementing the trigger algorithms for background rejection. A reduced selected stream is then addressed, through a $1 \mathrm{~Gb}$ Ethernet switch, from all the TCPUs to the Event Manager (EM) server which is deputed to write the trigger selected data on the local temporary storage device. Finally data are copied to the long lasting storage facility at the Laboratori Nazionali del Sud in Catania (Italy) by means of a dedicated $1 \mathrm{~Gb}$ Ethernet point to point connection.

\subsection{Positioning and calibration}

\subsubsection{Acoustic positioning system}

Since the tower structure is not rigid, floors are subject to the effects of deep-sea currents than can distort the vertical line shape of the tower, making the floors rotate and tilt. For a proper reconstruction of the muon tracks, which is based on space-time correlation of Cherenkov photons hitting the OMs, the knowledge of the position of each OM with a precision of the order of $10 \mathrm{~cm}$ is needed. This can be

\footnotetext{
${ }^{6}$ Xilinx Inc.
}

obtained by using a system based on acoustic triangulation as demonstrated by previous experience gained with the NEMO Phase-1 prototype [11].

The acoustic positioning system installed on the NEMO Phase- 2 tower consists of couples of hydrophones mounted in fixed positions close to the end of each floor $(\mathrm{H} 0$ and $\mathrm{H} 1$ in Fig. 6) [28,29]. For testing and validation purposes different types of hydrophones were used. The six lowermost floors are equipped with large broadband hydrophones $(10 \mathrm{~Hz}-70$ $\mathrm{kHz}) .^{7}$ A couple of free flooded rings (FFR) hydrophones ${ }^{8}$ is installed on the 7th floor. Finally, two of the OMs on the 8th floor are equipped with custom designed piezoelectric acoustic sensors glued to the internal surface of the glass sphere. Data from all acoustic receivers are sampled offshore at a frequency of $192 \mathrm{kHz}$ with a 24 bit resolution and continuously sent to shore. Offshore data are time stamped with the absolute acquisition time by a dedicated synchronous system which is phased with a GPS time station. ${ }^{9}$ The acoustic position system is completed with a set of autonomous acoustic beacons installed on the seafloor at a distance of approximately $400 \mathrm{~m}$ from the tower and one beacon at the base of the tower.

An independent real time monitoring of the floor orientation is provided by an Attitude Heading Reference System (AHRS), that includes triaxial gyroscopes, accelerometers and magnetometers, placed inside each FMPOD.

\subsubsection{Time calibration system}

The reconstruction of physical events (e.g., muon tracks) requires sub-nanosecond synchronisation between the OMs.

The synchronous communication protocol implemented on the tower provides synchronization with a unique clock source (Master Clock) for all electronics boards and OMs. The clock distribution system is based on a GPS station, which provides the absolute time, encoded in IRIG-B 1001344 standard, and a high-stability $10 \mathrm{MHz}$ clock which works as Master Clock for the detector [30].

Due to the clock propagation delay from shore to the tower, each OM has its own time offset to be measured and compensated. This is done using a fast light pulser installed in each $\mathrm{OM}$. The light pulsers are controlled by dedicated FPGA ${ }^{10}$ based control boards located in each FMPOD, which also allow setting the intensity and the frequency of the flashes of each light pulser. The system is operated from shore. The control boards can also perform measurements of the propagation delays of the commands to reach each of the light pulsers. The propagation delays have been proven to remain

\footnotetext{
7 Model SMID TR-401.

${ }^{8}$ Sensor Technology Ltd.

9 Symmetricom XLi.

10 Altera Cyclone III.
} 
stable down to the level of the resolution of the measurements, i.e. within 100 ps.

Prior to the deployment, a calibration of the OMs of the full tower has been performed by using an external system exploiting a fast laser source (Hamamatsu PLP-10) coupled to a network of optical fibres of calibrated lengths. Multimode, graded index optical fibres have been used for this application.

In order to have redundancy in time calibration, for exploring new solutions proposed for a $\mathrm{km}^{3}$-size detector as well as for measurements of the optical properties of water, the NEMO Phase-2 tower is also equipped with additional calibration devices comprising small LED pulsers, installed inside selected OMs in such a way to illuminate the OMs of the upper floors, and a laser beacon installed on the base of the tower.

\section{Installation and operation}

The NEMO Phase-2 tower was deployed on March 23 2013 during a sea campaign operated by the Multi Service Vessel 'Nautical Tide'. ${ }^{11}$ The tower deployment operations took approximately $6 \mathrm{~h}$. The tower was first lowered to the seabed and then connected to the CTF by means of a surface controlled ROV. After the connection the tower was unfurled reaching its final configuration. Data acquisition started immediately.

During the same operation one autonomous acoustic beacon was also deployed at about $400 \mathrm{~m}$ North of the tower. A second one was installed in a separate sea campaign on July 202013 at about $400 \mathrm{~m}$ East of the tower. Together with the one installed on the tower base they form the acoustic Long Baseline (LBL) system. These beacons were set to a repetition rate of $0.5 \mathrm{~Hz}$ and were not synchronized to the GPS clock distributed from shore. Their time of emission had to be re-synchronised with respect to the detector clock by means of the monitoring hydrophone mounted on the tower base in a fixed and known position. A malfunctioning in the power line cable of the monitoring hydrophones prevented the possibility of calibrating the LBL system. Consequently it was not possible to determine the position of each single hydrophone of the tower. However, information on the vertical position of the tower was provided by the CTD probes which were found to be at the expected mean depths of 3358 $\mathrm{m}$ (1st floor) and $3118 \mathrm{~m}$ (7th floor). This information was checked by measuring the time delays of acoustic signals, emitted by the beacon installed on the tower base, between the 1st floor and the other floors and found to be consistent with the CTD data.

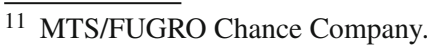

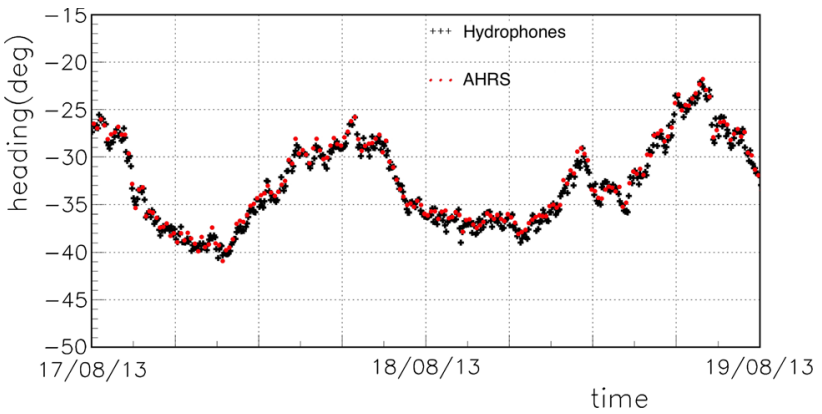

Fig. 9 Orientation of Floor 6 determined using the hydrophone data (black crosses) compared to the heading information determined by the AHRS installed on the Floor Control Module

Although in a reduced configuration, the acoustic data allow to recover the orientation (heading) and pitch and roll of each floor with a precision better than $1^{\circ}$. Using a global fit algorithm, the position of each hydrophone was calculated with an estimated error of about $1 \mathrm{~m}$. The acoustic data were combined with depth information from the two CTDs. A fixed distance between floors of $40 \mathrm{~m}$ and a $5 \mathrm{~m}$ uncertainty on the position of the LBL beacons, determined by the ship GPS system at the time of deployment, were assumed. As an example, a floor heading reconstruction by using the hydrophones system is shown in Fig. 9 for period of two days. This is in excellent agreement with the AHRS heading reconstruction (15 min averages) normalized to the hydrophone data.

Despite the lack of a precise positioning of each single $\mathrm{OM}$ it was however possible to reconstruct downgoing muon tracks and determine the Depth Intensity Relation of muons in water as reported in [31].

In a limited number of runs a new test device, i.e. a beacon located at the tower base whose emission is synchronous with the master clock, was used. In this case it was possible to reduce the effect of systematic errors and determine the position of each hydrophone with an accuracy of about 30 cm comparable to those obtainable with a complete LBL.

The commissioning phase of the tower lasted up to 30 June 2013. During this phase the PMT gains were adjusted several times to obtain the same gain factor of $5 \times 10^{7}$. This converts each single photon electron (s.p.e.) into a mean integrated charge of about $8 \mathrm{pC}$. Thresholds were adjusted at approximately 0.25 s.p.e. As a consequence data rates reported in the following have to be considered stable only after the completion of this phase.

Trigger used during the data taking are described in [31].

\section{Long term monitoring of the optical background}

During seventeen months of operation the NEMO Phase-2 tower allowed for a continuous long term monitoring of the 


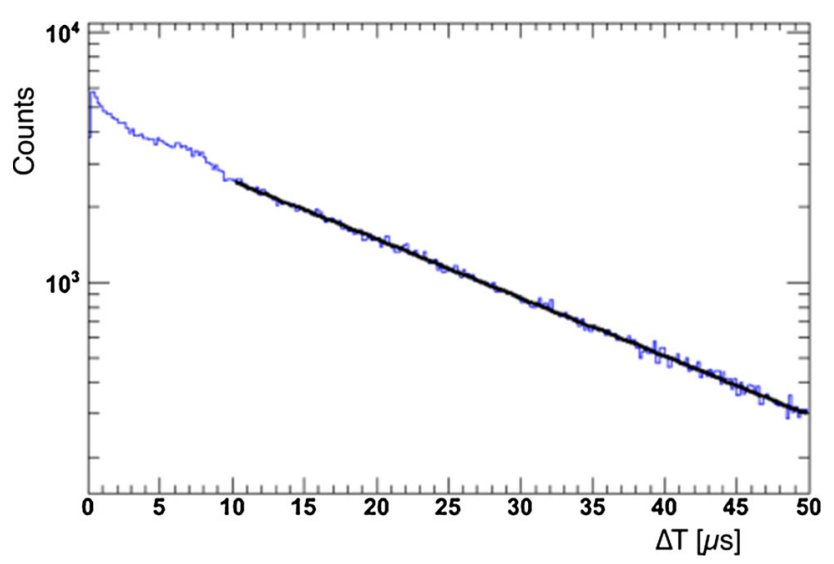

Fig. 10 Time difference between two consecutive hits. The exponential fit above $\Delta t=10 \mu \mathrm{s}$ (black line) gives a characteristic time $\tau=1.8 \times 10^{-5} \mathrm{~s}$. The bump at $\Delta t \approx 7 \mu \mathrm{s}$ is due to the presence of PMT afterpulses. For $\Delta t<1 \mu \mathrm{s}$ the effect of electronics deadtime is present

site characteristics. In particular, the optical background rate, due to the presence of Cherenkov light produced in the decay of ${ }^{40} \mathrm{~K}$ as well as light emitted by bioluminescent organisms, was studied.

The distribution of the time difference between successive hits, $\Delta t$, of a typical OM, is shown in Fig. 10. It has the expected exponential form, with a slope of $\tau=1.8 \times 10^{-5}$ $\mathrm{s}$ corresponding to a purely random background singles rate of about $55 \mathrm{kHz}$.

The expected singles rate has been evaluated by using a complete GEANT4 simulation, that accounts for the ${ }^{40} \mathrm{~K}$ contribution, the PMT dark counts and the presence of radioactive materials in the glass sphere [32]. The two latter were measured in the laboratory. The water absorption length of the KM3NeT-It site has been used [13]. The simulation also takes into account the shadowing effects of the mechanical structure holding the OMs. The result of this simulation gives a value of $54 \pm 3 \mathrm{kHz}$ in agreement with the in situ observed rate. Optical module relative efficiencies were measured in the laboratory on a set of six optical modules and used as input to tune the Monte Carlo simulations. The major source of uncertainty on the simulated singles rate comes from the spread of the quantum efficiencies in the photocatodes examined [32] and can be considered as representative of the full set of PMTs used in the NEMO Phase- 2 tower. The standard deviation of these measurements was taken into account and propagated to the simulation results.

Double coincidences, originating from ${ }^{40} \mathrm{~K}$ decays, between close neighbour OMs have also been studied. As an example for the two OM couples at the extremities of the 1st floor, values of $20.9 \pm 0.9 \mathrm{~Hz}$ and $21.5 \pm 0.8 \mathrm{~Hz}$ were measured. For the other OM couples the values range from 19.0 to $22.5 \mathrm{~Hz}$ with an average value of $20.45 \pm 1.5 \mathrm{~Hz}$. The spread can be mainly ascribed to differences in the PMT threshold
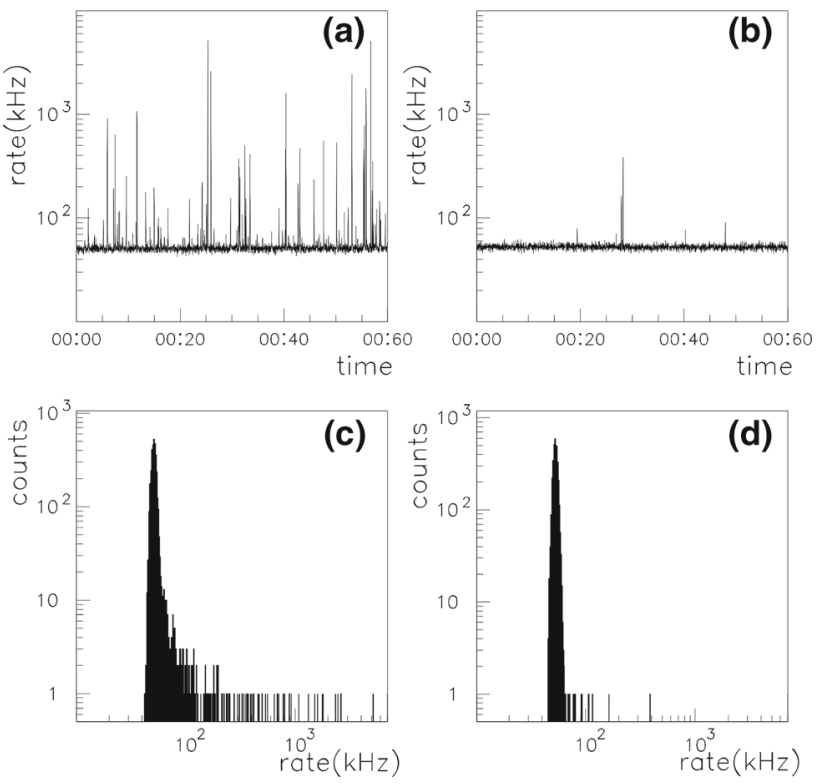

Fig. 11 Singles rate as measured in the monitoring channel as a function of time for $1 \mathrm{~h}$ time slots measured on July 112013 (panel $a$ ) and on September 112013 (panel b). Panels $c, d$ show the corresponding histograms of the monitored rate values

and on the photocatode quantum efficiency. For OM couples with the same geometry the Monte Carlo simulations gives a value of $21.6 \mathrm{~Hz}$ [32] in good agreement with the measured data.

The rates determined as in Fig. 10 on the acquired data suffer from the dead time introduced by the data acquisition system that limits the maximum data rate transferable to about $150 \mathrm{kHz}$. However, the FEM board on each OM provides an independent monitoring of the average count rate by counting once per second the number of hits with amplitude exceeding a threshold of $\approx 0.25$ s.p.e. in a $10 \mathrm{~ms}$ time window. This estimate does not suffer from dead time limitations allowing to monitor the signal rate up to about 6.5 $\mathrm{MHz}$. Figure 11 shows the distribution of the singles rates (measured by the OM rate monitor) in one OM for two 1$\mathrm{h}$ time slots taken in different periods with different optical background characteristics. In the top panels ( $a$ and $b$ ) the rate is shown as a function of time. In the bottom panels ( $\mathrm{c}$ and d) the corresponding histograms of the rate distributions are shown. In general, the instantaneous rate exhibits a flat baseline at around $55 \mathrm{kHz}$ with some sporadic bursts. The baseline corresponds to the contribution of the ${ }^{40} \mathrm{~K}$ discussed above while light bursts can be attributed to the presence of bioluminescent organisms. The amount of these bursts can vary in time, as shown by the two examples in Fig. 11. To monitor these variations, as well as possible variations in the baseline rate, histograms like those of Fig. 11c, d have been built for 15 min-long time frames covering the whole operation period. From each histogram the baseline rate was extracted 


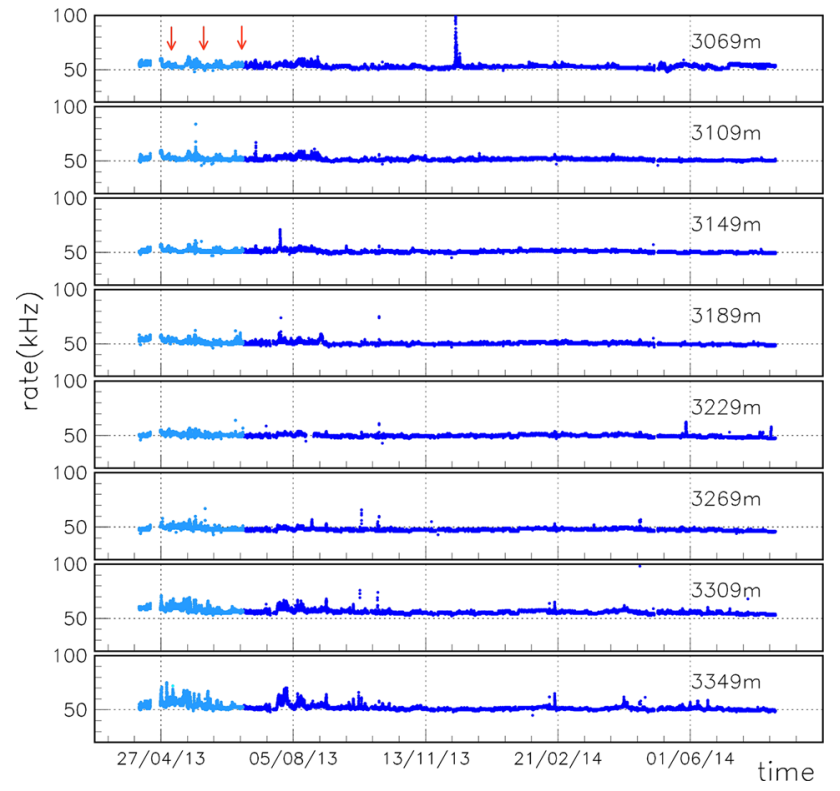

Fig. 12 Baseline rate measured for the whole operation period of the tower at different depths. All selected PMTs are down-looking. Light blue dots correspond to the commissioning period of the detector. The arrows indicate the dates when PMT high voltage adjustments were performed

as median of the distribution. The amount of light bursts was determined as the fraction of time with rate exceeding an arbitrarily fixed threshold of $100 \mathrm{kHz}$ (burst fraction).

In Fig. 12, the baseline rate for eight OMs, all chosen with the same down-looking orientation and located at different depths, is shown for the whole operation period of the tower. For the sake of completeness the data taken during the commissioning period are also shown (light blue dots). The observed baseline rates do not show large fluctuation above $50-60 \mathrm{kHz}$ and are in agreement with previous measurements performed in the same site. Sporadic highbaseline rates, which are however always below $100 \mathrm{kHz}$, are observed. They can be attributed to periods with a higher level of bioluminescent activity. The average value for the whole measurement period and for all floors is compatible with the estimate of the ${ }^{40} \mathrm{~K}$ decay contribution. No significant effect due to the depth is observed. The measured rates are in general lower and more stable than those observed in the ANTARES neutrino detector site [1] and reported in [33].

For a comparison between optical modules at the same depth but with different orientation, the baseline rates for the for OMs of the 1 st floor of the tower are shown in Fig. 13. Also in this case the rates are similar. However, a slight decrease with time of the rates measured with the two horizontally looking OMs can be observed. The origin of this is unknown but can be reasonably attributed to the deposition of a thin sediment layer on the uppermost part of the optical module.

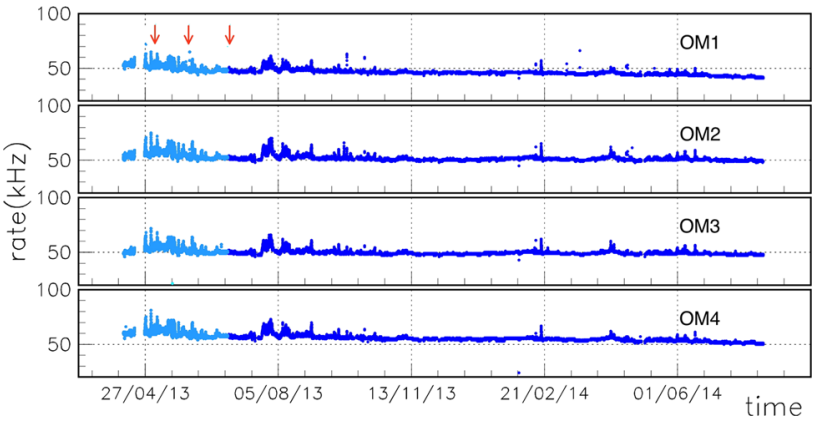

Fig. 13 Baseline rate measured for the whole operation period of the tower for the four OMs of the 1st floor of the tower. The OM numbering follows the scheme illustrated in Fig. 6 with OM 1 and 4 that are horizontally looking and OM 2 and 3 downward looking. Light blue dots correspond to the commissioning period of the detector. The arrows indicate the dates when PMT high voltage adjustments were performed

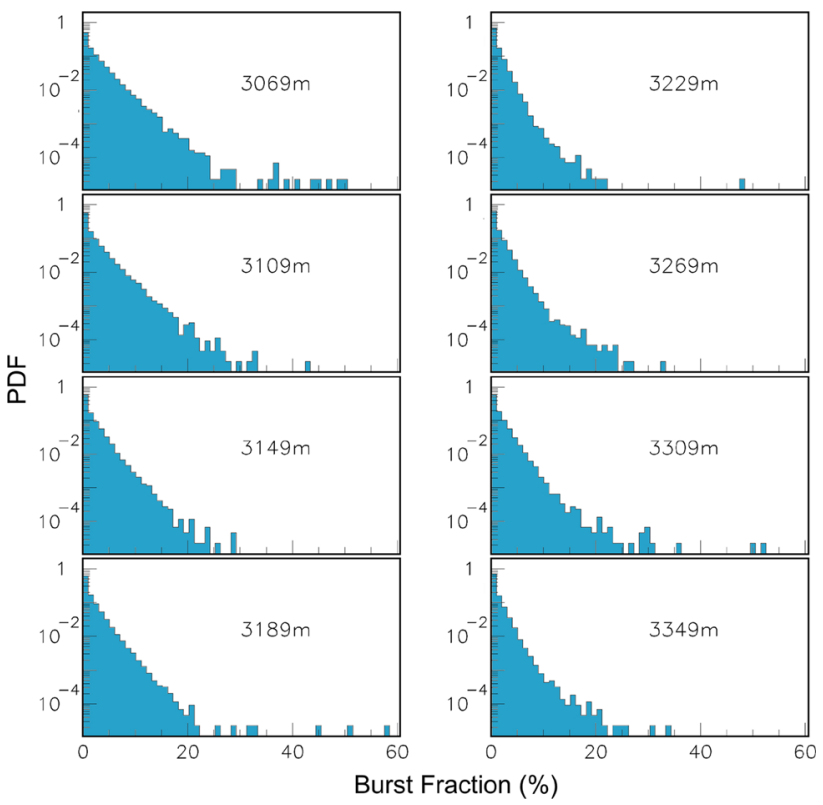

Fig. 14 Probability Density Function (PDF) of observing a given burst fraction, defined as having a rate exceeding $100 \mathrm{kHz}$ in a $10 \mathrm{~ms}$ time window, in eight down-looking OMs placed at different depths

For the same set of eight down-looking OMs the Probability Density Function of having a rate exceeding $100 \mathrm{kHz}$ in a $10 \mathrm{~ms}$ time interval are reported in Fig. 14. No significant depth dependence is observed. This effect can be seen also in Fig. 15 (upper panel) where the Probability Density Function of the rate has been calculated after the last $\mathrm{HV}$ adjustment for the period going from July 2013 until August 2014. In particular, the contribution of bioluminescence bursts is shown for an optical module located on the 1 st floor of the tower. For the examined $\mathrm{OM}$, the rate distribution is peaked around 50 $\mathrm{kHz}$ and becomes negligible above $100 \mathrm{kHz}$. The other optical modules show very similar behaviors with peaks located in the range $45-55 \mathrm{kHz}$. The Cumulative Density Function of the rate has also been calculated and it reaches the value of 

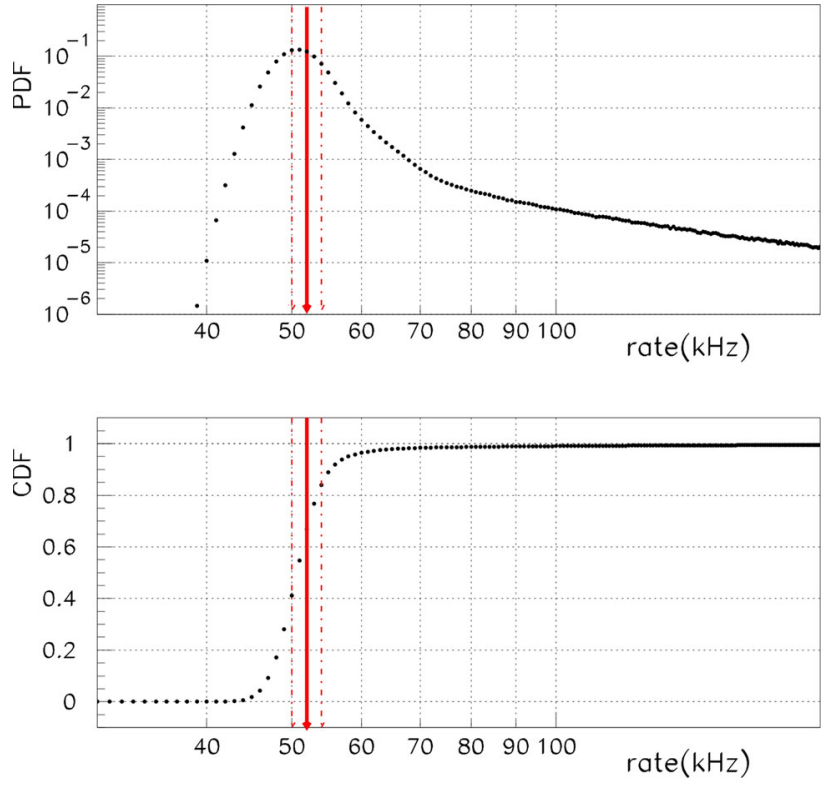

Fig. 15 Probability Density Function (PDF) and Cumulative Density Function (CDF) of singles rate (black dots) for the whole set of data taken from July 2013 to August 2014 of one PMT. The solid red line indicates the result of the GEANT4 simulation described in the text. Dashed red lines give the error in this simulation

0.99 in the range $100-200 \mathrm{kHz}$. This leads to the important conclusion that the probability to have the TriDAS in dead time is less than $1 \%$ all along the full year observation. There is no period in which the OMs needed to be switched off for high bioluminescence activity.

For a better evaluation of the impact of bioluminescent bursts on the detector performance a study of the coincidences between bursts on OMs on the same floor has been carried out. For a visual comprehension a sample snapshot of $1000 \mathrm{~s}$, recorded during a period of high level bioluminescence, is shown in Fig. 16. The four panels show the time series of the instant rates measured on the four OMs of the 1st floor. The OM numbering follows the scheme illustrated in Fig. 6. A quantitative analysis has been performed by evaluating over the whole data sample the probability $P_{i}$ of having a rate exceeding $100 \mathrm{kHz}$ in a single $\mathrm{OM}$ and the probability $P_{i j}$ of having a rate exceeding $100 \mathrm{kHz}$ simultaneously in a pair of OMs. This has been computed for all the six possible pairs of OMs of the 1st floor and reported in Table 1. The correlation factor, defined as $r_{i j}=\left(P_{i j}-P_{i} \times P_{j}\right) / \sqrt{\left(P_{i}-P_{i}^{2}\right)\left(P_{j}-P_{j}^{2}\right)}$ is also reported. Close-by OMs (pair 1,2 and 3,4) and the two downward looking OMs (pair 2,3), which observe the same volume of water, show a higher rate of burst coincidences with a higher degree of correlation. However, the probability of observing the same burst simultaneously in both OMs is always less than $0.2 \%$. For distant OMs the probability of

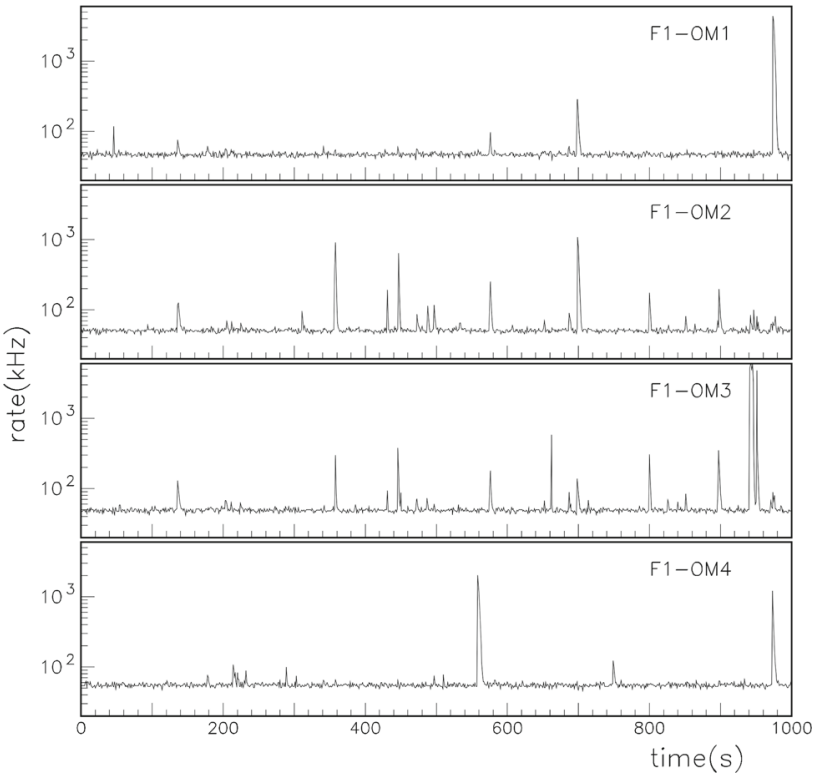

Fig. 16 Singles rate as measured in the monitoring channel of the four OMs of the 1st floor as a function of time for a $1000 \mathrm{~s} \mathrm{time} \mathrm{slot} \mathrm{mea-}$ sured on July 112013 , corresponding to a period of relatively high-level bioluminescence activity (same as the one shown in Fig. 11a)

Table 1 Probabilities $P_{i}$ and $P_{j}$ to observe independently a rate exceeding $100 \mathrm{kHz}$ in a pair $(i, j)$ of OMs compared with the probability $P_{i j}$ to observe a burst exceeding $100 \mathrm{kHz}$ simultaneously in both OMs

\begin{tabular}{lllll}
\hline OM pair & $P_{i}$ & $P_{j}$ & $P_{i j}$ & $r_{i j}$ \\
\hline$(1,2)$ & $5.03 \times 10^{-3}$ & $1.09 \times 10^{-2}$ & $2.55 \times 10^{-3}$ & 0.34 \\
$(3,4)$ & $9.30 \times 10^{-3}$ & $5.71 \times 10^{-3}$ & $2.67 \times 10^{-3}$ & 0.36 \\
$(1,3)$ & $5.03 \times 10^{-3}$ & $9.30 \times 10^{-3}$ & $1.38 \times 10^{-3}$ & 0.20 \\
$(2,4)$ & $1.09 \times 10^{-2}$ & $5.71 \times 10^{-3}$ & $1.46 \times 10^{-3}$ & 0.18 \\
$(2,3)$ & $1.09 \times 10^{-2}$ & $9.30 \times 10^{-3}$ & $4.33 \times 10^{-3}$ & 0.42 \\
$(1,4)$ & $5.03 \times 10^{-3}$ & $5.71 \times 10^{-3}$ & $2.70 \times 10^{-4}$ & 0.05 \\
\hline
\end{tabular}

A correlation factor $r_{i j}$ (see text) is also reported. The data refers to the six possible pairs of OMs of the 1st floor

simultaneously observing the same burst is of the order of $10^{-3}$ or less with a lower degree of correlation.

The time behaviour of the small bioluminescence activity is shown in Fig. 17 for a 15 days time series. There is evidence of periodic behaviour due to the presence of inertial currents which at the tower latitudes have a period of $20.21 \mathrm{~h}$. Further analyses of bioluminescence correlation with sea currents are in progress.

\section{Conclusions and perspectives}

Deployed in March 2013 in the abyssal site of Capo Passero at the depth of $3500 \mathrm{~m}$, the NEMO Phase- 2 tower continuously took data until August 2014, validating the technical solutions proposed for the construction of an under- 


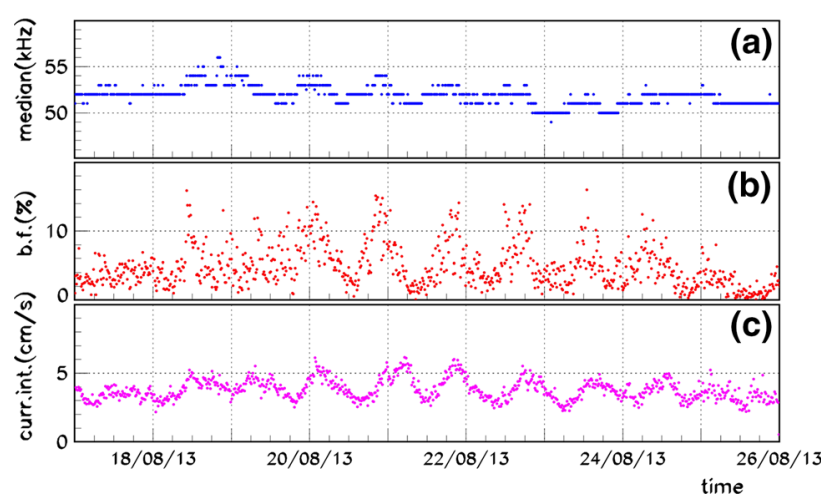

Fig. 17 a Baseline rates over a 15 min period; b burst fraction (b.f.), defined as the fraction of time in the $15 \mathrm{~min}$ window with rate exceeding $100 \mathrm{kHz}$; c modulus of the water current velocity measured with the DCS

water Cherenkov neutrino detector. The data analyses have confirmed the optimal environmental characteristics of the KM3NeT-It deep-sea site.

This site will host the Italian node of the KM3NeT underwater neutrino telescope. This detector will comprise eight tower-like detection units, built with a similar technology as the one used for the NEMO Phase-2 prototype described here but with 14 floors each one holding six optical modules, and 24 string-like detection units, using a new technology developed by the European KM3NeT collaboration.

Open Access This article is distributed under the terms of the Creative Commons Attribution 4.0 International License (http://creativecomm ons.org/licenses/by/4.0/), which permits unrestricted use, distribution, and reproduction in any medium, provided you give appropriate credit to the original author(s) and the source, provide a link to the Creative Commons license, and indicate if changes were made.

Funded by SCOAP ${ }^{3}$.

\section{References}

1. M. Ageron et al., ANTARES: the first undersea neutrino telescope. Nucl. Instr. Methods A 656, 11 (2011)

2. V. Aynutdnov for the Baikal Coll., The BAIKAL neutrino project: results and perspective. Nucl. Instr. Methods. A 628, 115 (2011)

3. A. Achterberg et al., First year performance of the IceCube neutrino telescope. Astropart. Phys. 26, 155 (2006)

4. M.G. Aartsen et al., Evidence for high-energy extraterrestrial neutrinos at the IceCube detector. Science 342, 1242856 (2013)

5. M.G. Aartsen et al., Observation of high-energy astrophysical neutrinos in 3 years of IceCube data. Phys. Rev. Lett. 113, 101101 (2014)

6. M.G. Aartsen et al., Evidence for astrophysical muon neutrinos from the northern sky with IceCube. Phys. Rev. Lett. 115, 081102 (2015)

7. E. Migneco et al., Status of NEMO. Nucl. Instr. Methods A 567, 444 (2006)

8. E. Migneco et al., Recent achievements of the NEMO project. Nucl. Instr. Methods A 588, 111 (2008)
9. A. Capone et al., Recent results and perspectives od the NEMO project. Nucl. Instr. Methods A 602, 47 (2009)

10. M. Taiuti et al., The NEMO project: a status report. Nucl. Instr. Methods A 626, S25 (2011)

11. S. Aiello et al., Measurement of the atmospheric muon flux of the NEMO Phase-1 detector. Astropart. Phys. 33, 263 (2010)

12. A. Capone et al., Measurements of light transmission in deep sea with the AC9 transmissometer. Nucl. Instr. Methods A 487, 423 (2002)

13. G. Riccobene et al., Deep seawater inherent optical properties in the Southern Ionian Sea. Astropart. Phys. 27, 1 (2007)

14. A. Rubino et al., Abyssal undular vortices in the Eastern Mediterranean basin. Nat. Commun. 3, 834 (2012)

15. KM3NeT web site. www.km3net.org

16. M. Sedita for the NEMO collaboration, Electro-optical cable and power feeding system for the NEMO Phase-2 project. Nucl. Instr. Methods A 567, 531 (2006)

17. R. Cocimano for the NEMO collaboration, A comparison of $\mathrm{AC}$ and DC power feeding systems based on the NEMO experiences. Nucl. Instr. Methods A 602, 171 (2009)

18. A. Orlando for the NEMO collaboration, On line monitoring of the power control and engineering parameters systems of the NEMO Phase-2 tower. Nucl. Instr. Methods. A 602, 180 (2009)

19. M. Musumeci for the NEMO collaboration, Construction and deployment issues for $\mathrm{km}^{3}$ underwater detector. Nucl. Instr. Methods. A 567, 545 (2006)

20. S. Aiello et al., The optical modules of the phase- 2 of the NEMO project. JINST 8, P07001 (2013)

21. E. Leonora, S. Aiello, Design and assembly of the optical modules for phase- 2 of the NEMO project. Nucl. Instr. Methods A 725, 234 (2013)

22. S. Aiello et al., Procedures and results of the measurements on large area photomultipliers for the NEMO project. Nucl. Instr. Methods A 614, 206 (2010)

23. C.A. Nicolau for the NEMO collaboration, An FPGA-based readout electronics for neutrino telescopes. Nucl. Instr. Methods A 567, 552 (2006)

24. M. Cordelli et al., PORFIDO: oceanographic data for neutrino telescopes. Nucl. Instr. Methods A 626-627, S109 (2011)

25. F. Ameli, The data acquisition and transport design for NEMO Phase-1. IEEE Trans. Nucl. Sci. 55(1), 233 (2008)

26. A. D'Amico for the NEMO collaboration, Design of the optical Raman amplifier for the shore station of NEMO Phase-2. Nucl. Instr. Methods A 626-627, S173 (2011)

27. T. Chiarusi for the NEMO collaboration, Scalable TriDAS for the NEMO project. Nucl. Instr. Methods A 630, 107 (2011)

28. S. Viola et al., NEMO-SMO acoustic array: a deep-sea test of a novel acoustic positioning system for $\mathrm{a} \mathrm{km}^{3}$-scale underwater neutrino telescope. Nucl. Instr. Methods A 725, 207 (2013)

29. S. Viola et al., in Underwater acoustic positioning system for the SMO and KM3NeT-Italia projects. AIP Conference Proceedings 1630, 134 (2014)

30. M. Circella for the NEMO collaboration, Time calibration of the NEutrino Mediterranean Observatory (NEMO). Nucl. Instr. Methods A 602, 187 (2009)

31. S. Aiello et al., Measurement of the atmospheric muon depth intensity relation with the NEMO phase- 2 tower. Astropart. Phys. 66, 1 (2015)

32. C. Hugon for the ANTARES and KM3NeT collaborations, Step by step simulation of phototubes for the KM3NeT and ANTARES optical modules. Nucl. Instr. Methods A 787, 189 (2015)

33. Ch. Tamburini et al., Deep-sea bioluminescence blooms after dense water formation at the ocean surface. PLOS One 8, e67523 (2013) 